\title{
Diagnostic characterization of respiratory allergies by means of a multiplex immunoassay
}

\author{
J. L. M. Millen, ${ }^{* \dagger}$ I. Willems, ${ }^{* \dagger}$ \\ G. Slingers, ${ }^{\star \star \S}$ M. Raes, ${ }^{\ddagger \S}$ G. Koppen ${ }^{\star}$ \\ and S. A. S. Langie (iD *tg \\ ${ }^{*}$ VITO - Health, Mol, ${ }^{\dagger}$ Centre for \\ Environmental Sciences, Hasselt University, \\ ${ }^{\ddagger}$ Faculty of Medicine and Life \\ Sciences, UHasselt, LCRC, Diepenbeek, \\ ${ }^{\lessgtr}$ Paediatrics, Jessa Hospital, Hasselt, and \\ Department of Pharmacology and \\ Toxicology, School for Nutrition and \\ Translational Research in Metabolism
}

(NUTRIM), Maastricht University, Maastricht, the Netherlands

\begin{abstract}
Summary
Allergic sensitization is commonly assessed in patients by performing the skin prick test (SPT) or determining specific immunoglobulin (IgE) levels in blood samples with the ImmunoCAP ${ }^{\mathrm{m} w}$ assay, which measures each allergen and sample separately. This paper explores the possibility to investigate respiratory allergies with a high throughput method, the Meso Scale Discovery (MSD) multiplex immunoassay, measuring IgE levels in low volumes of blood. The MSD multiplex immunoassay, developed and optimized with standards and allergens from Radim Diagnostics, was validated against the SPT and the ImmunoCAP assay. For 18 adults (15 respiratory allergy patients and three controls), blood collection and the SPT were performed within the same hour. Pearson correlations and Bland-Altman analysis showed high comparability of the MSD multiplex immunoassay with the SPT and the ImmunoCAP assay, except for house dust mite. The sensitivity of the MSD multiplexed assay was $\geq 78 \%$ for most allergens compared to the SPT and ImmunoCAP assay. Additionally, the specificity of the MSD multiplex immunoassay was $\geq 87 \%$ - the majority showing $100 \%$ specificity. Only the rye allergen had a low specificity when compared to the SPT, probably due to cross-reactivity. The reproducibility of the MSD multiplex immunoassay, assessed as intra- and interassay reproducibility and biological variability between different sampling moments, showed significantly high correlations $(r=0.943-1)$ for all tested subjects (apart from subject 13; $r=0 \cdot 65-0.99$ ). The MSD multiplex immunoassay is a reliable method to detect specific IgE levels against respiratory allergens in a multiplexed and high-throughput manner, using blood samples as small as from a finger prick.
\end{abstract}

Keywords: allergens, high throughput, immunoglobulin E, multiplex immunoassay, respiratory allergies

\section{Introduction}

Respiratory allergies are the most common allergies worldwide, still increasing in frequency and severity. The World Health Organization estimated patients suffering from allergic asthma at 235 million in 2013 and the numbers for allergic rhinitis at about 400 million in 2006 [1,2]. Although the number of patients with respiratory allergy drastically increased on a global scale, the increase is greatest in western countries. Blomme et al. performed the skin prick test (SPT) on 2320 Flemish participants (aged 3-86 years) and reported a $40 \cdot 3 \%$ prevalence of a positive SPT to one or more common aeroallergens and clinical allergic rhinitis symptoms in $30.9 \%$ of cases [3]. The European Academy of Allergy and Clinical Immunology (EAACI) reported in 2015 that up to $40 \%$ of the population was affected by allergic rhinitis worldwide, with the highest prevalence in the developed nations of the northern Hemisphere, $23-30 \%$ affected population in Europe and $12-30 \%$ in the United States [4]. Notably, a vast majority of individuals affected by allergies are children and young adults [5]. To emphasize the 
increasing prevalence, according to the Belgian Health Status Report the self-reported allergy prevalence in the Belgian population older than 15 years was $13.3 \%$ in 2008 , $14 \cdot 2 \%$ in 2013 and $18 \cdot 7 \%$ in 2018 [6]. Although allergic rhinitis is not being associated with high morbidity, allergic asthma is. In addition, both types of respiratory allergy have generated a burden on society due to both the impact on the quality of life and the cost of treatment. A quick and precise diagnosis to identify the causative allergens is of fundamental importance to establish the most adequate treatment [7].

In addition to the history and the clinical examination, looking for immunoglobulin (IgE) sensitization is an essential step in the diagnosis of complaints compatible with allergy. The data suggest that more than 50\% of allergic patients are multi-sensitized, making diagnosis even more challenging [8]. Traditionally, two methods are applied: in vivo by executing an SPT or in vitro by the detection of sIgE in blood. There are several types of allergy blood tests, among which the radioallergosorbent test (RAST) was originally the preferred blood test, but has been replaced by more sensitive fluorescence enzyme-labelled assays [9]. This study used specifically the ImmunoCAP methodology, as this assay is currently most commonly used as allergy test in clinical laboratories [10-12]. The SPT is quick and results are immediately known; however, it requires trained clinical personnel to perform it. Although the SPT is sensitive, minimally invasive and cost-effective, in-vitro methods such as ImmunoCAP and enzyme-linked immunosorbent assay (ELISA) have some advantages, including direct quantitation and the possibility of long-term storage of specimens. Notwithstanding, the in-vitro procedures present limitations that are an impediment for large studies and for prospective studies to be performed over several years. For instance, measurements for each allergen and sample has to be performed separately, which is timeconsuming and is, therefore, associated with higher costs and increased possibility for technical errors. Comparing studies of the two above-mentioned test methods, the SPT seems to be more sensitive (fewer false-negative results), while sIgE immunoassays seem to be more specific (fewer false-positive results) [13,14].

During the last decade, there was a gradual evolution from performing singleplex towards multiplex immunoassays. These multiplex immunoassays provide several advantages over singleplex immunoassays, including increased efficiency at reduced expense, lower sample volume needed, making it interesting from a pediatric standpoint, greater output (number of markers assessed) per sample volume and higher throughput predicting more detailed diagnostics, thereby facilitating personalized medicine [15]. Currently, various singleplex as well as multiplex immunoassays are available to investigate respiratory allergy-associated immunological protein markers [e.g. by Luminex, Fireplex, Meso Scale Discovery (MSD)]. In this study, we utilized the MSD (Meso Scale Diagnostics, Rockville, MD, USA) multiplex immunoassays, considering that this method ensures all the abovementioned advantages, with only $25 \mu \mathrm{l}$ of serum volume required for analysis. This particular advantage cannot be emphasized enough, as taking blood samples from children remains a demanding technique and is an ethically sensitive issue. Using the MSD multiplex immunoassay, a finger prick could be sufficient to collect enough sample volume to conduct the test. Similarly, biomonitoring studies could also benefit from this advantage. Additionally, the MSD multiplex immunoassay is a highly flexible method, as the allergen panel can be changed according to the individual needs of the patient. Both individual allergens and allergen mixtures could be spotted on either a complete or partial 96-well plate, making the test even more compelling for either individual diagnosis or biomonitoring studies. Novel techniques, such as the ImmunoCAP Rapid point-of-care, which can be performed on children and adults, still require $110 \mu \mathrm{l}$ of sample volume [16,17]. Moreover, this technique is not flexible, as it operates via a fixed allergen panel. Another recent multiplex technique is the Immuno Solid-phase Allergen Chip (ISAC) assay, based on microchip technology $[11,18]$. The major disadvantages of the ISAC assay include semi-quantitative results and the requirement of $4 \mathrm{~h}$ of fasting. A comparison between the ImmunoCAP (Rapid) assay, ISAC test and the MSD multiplex immunoassay is shown in Supporting information, Table S1.

To date, validation of multiplex immunoassays for in-vitro testing in clinical settings is limited. In this regard, this study aimed to investigate the possibility of diagnosing respiratory allergies with the MSD multiplex immunoassay while simultaneously comparing this method to both an SPT and an ImmunoCAP assay. Both single allergen extracts as well as allergen mixes were tested in the MSD multiplex immunoassay, as the use of allergen mixes might be interesting for quick screening purposes in biomonitoring or molecular epidemiological studies. Statistical analysis demonstrated high comparability of the MSD multiplex immunoassay and the ImmunoCAP assay. Both tests appear reliable in terms of sensitivity and specificity. Moreover, the reproducibility of the MSD multiplex immunoassay was determined, including intra- and interassay variation and reproducibility over time between different sampling moments. The reproducibility parameters established significantly high Pearson correlations. Finally, a shorter version of the protocol, including $2 \mathrm{~h}$ instead of overnight incubation with the samples, provides results within $8 \mathrm{~h}$ after blood collection. 


\section{Materials and methods}

\section{Study population}

The study population consisted of 18 adults (12 females and six males; age range $=23-50$ years; mean $=34 \cdot 8 \pm$ 8.2 years). Three non-allergic controls and 15 patients were included. Patients were selected and enrolled based on their self-reported and/or doctor's diagnosed allergy symptoms. More specifically, 14 participants reported clinical symptoms of hay fever, including 12 participants who suffered from tree pollen allergy, five were allergic to cats and dogs, while three were only allergic to cats. Moreover, nine of these 14 patients reported allergy symptoms on exposure to house dust mite, and one person reported allergic asthma in reaction to pollen and house dust mite. In addition, four reported allergy against hazelnut (including one against almonds and two against walnuts) ever or in the last 12 months, and one person reported allergy against cow's milk. The 15th patient (subject 3) reported no clinical symptoms in reaction to respiratory allergens, but indicated suffering from sun allergy (i.e. reaction to ultraviolet radiation) and food allergy (i.e. banana). Questions to gather the information on clinical allergy symptoms were based on the ISAAC questionnaire [19]. Sampling was performed in August. All subjects were asked to stop all anti-allergic medication for at least a week before sampling. Written informed consent was obtained from all individuals and the study was approved by the medical ethics committee UZA-UA (EC UZA 18/11/154).

\section{SPT}

Droplets of allergens, histamine (positive control) and a negative control were applied to the forearms of the subjects. In each droplet, a small prick was made into the skin. Skin reactions caused by the allergens were determined after $15 \mathrm{~min}$ by a medical doctor. The size (wheal: length and width) of redness (flare) on the skin around the allergens and controls was measured. A wheal reaction of at least $3 \mathrm{~mm}$ in size compared to the negative control was used as a cut-off to classify the test as positive. The allergens used in this analysis are shown in Table 1; $\mathrm{d}$ 1, e1, e5, t3, w6 and 'spring trees mix I' allergen extracts were purchased from HAL-Allergy Benelux BV (Leiden, the Netherlands) and $\mathrm{f} 2, \mathrm{~g} 6, \mathrm{~g} 12, \mathrm{~m} 2$ were purchased from ALK-ABELLO BV (Almere, the Netherlands).

\section{Blood analysis}

From each individual, two blood serum tubes of $10 \mathrm{ml}$ were collected. One serum tube was sent to a clinical laboratory (Labo Rigo, Genk, Belgium), where the

Table 1. Overview of the allergens used in the different tests

\begin{tabular}{|c|c|c|c|}
\hline Allergens (allergen number)* & Skin prick test & ImmunoCAP & MSD multiplex immunoassay \\
\hline Dust mite Dermatophagoides pteronyssinus (d1) & $\mathrm{X}$ & $\mathrm{X}$ & $\mathrm{X}$ \\
\hline Cat epithelium (e1) & $\mathrm{X}$ & $\mathrm{X}$ & $\mathrm{X}$ \\
\hline Dog hair and dander (e5) & $\mathrm{X}$ & $\mathrm{X}$ & $\mathrm{X}$ \\
\hline Cow’s milk (f2) & $\mathrm{X}$ & $\mathrm{X}$ & $\mathrm{X}$ \\
\hline Rye; Secale cereale (g12) & $\mathrm{X}$ & $\mathrm{X}$ & $\mathrm{X}$ \\
\hline Timothy grass; Phleum pratense (g6) & $\mathrm{X}$ & $\mathrm{X}$ & $\mathrm{X}$ \\
\hline Cladosporium herbarum (m2) & $\mathrm{X}$ & $\mathrm{X}$ & $\mathrm{X}$ \\
\hline Birch; Betula verrucosa $(\mathrm{t} 3)$ & & $\mathrm{X}$ & $\mathrm{X}$ \\
\hline Mugwort; Artemisia vulgaris (w6) & $\mathrm{X}$ & $\mathrm{X}$ & $\mathrm{X}$ \\
\hline Mixed animals (ex1): cat (e1), dog (e2), horse (e3) and cow (e4) & & & $\mathrm{X}$ \\
\hline $\begin{array}{l}\text { Mixed nuts (fx1): peanut (f13), walnut (f16), hazelnut (f17), almond (f20) and } \\
\text { coconut (f36) }\end{array}$ & & & $\mathrm{X}$ \\
\hline $\begin{array}{l}\text { Mixed grasses (gx3): sweet vernal grass (g1), perennial ryegrass (g5), timothy } \\
\text { grass (g6), rye (g12) and common velvet grass (g13) }\end{array}$ & & $\mathrm{X}$ & $\mathrm{X}$ \\
\hline $\begin{array}{l}\text { House mix (hx1): house dust (h1), dust mite Dermatophagoides pteronyssinus } \\
\text { (d1), dust mite Dermatophagoides farina (d2) and cockroach (i6) }\end{array}$ & & & $\mathrm{X}$ \\
\hline $\begin{array}{l}\text { Mixed fungi (mx1): Penicillium notatum }(\mathrm{m} 1) \text {, Cladosporium herbarum }(\mathrm{m} 2) \text {, } \\
\text { Aspergillus fumigatus }(\mathrm{m} 3) \text { and Alternaria alternate }(\mathrm{m} 6)\end{array}$ & & $\mathrm{X}$ & $\mathrm{X}$ \\
\hline Mixed trees (tx2): ${ }^{\dagger}$ hazel (t4), birch (t3), alder (t2) and ash (t15) & $\mathrm{X}$ & $\mathrm{X}$ & $\mathrm{X}$ \\
\hline $\begin{array}{l}\text { Mixed weeds (wx2): Alsem ambriosa (w1), mugwort (w6), daisy (w7), dandelion } \\
\text { (w8) and narrow-leaf plantain (w9) }\end{array}$ & & & $\mathrm{X}$ \\
\hline
\end{tabular}

Allergen extracts for the skin prick test were purchased from HAL-Allergy Benelux BV (Leiden, the Netherlands; d1, e1, e5, t3, w6 and 'spring trees mix I') and from ALK-ABELLO BV (Almere, the Netherlands; f2, g6, g12, m2). MSD = Meso Scale Discovery.

${ }^{*}$ Allergen codes are according to RADIM Diagnostics.

${ }^{\dagger}$ The allergen code was different for the ImmunoCAP (Thermo Scientific) analysis by the medical laboratory (tx10). 
ImmunoCAP assay from Thermo Fisher Scientific (Waltham, MA, USA) was performed to measure the total $\operatorname{IgE}$ level and specific IgE levels for respiratory allergens, according to the manufacturer's protocol. The allergens analysed in this assay are shown in Table 1. The blood serum of the second tube was isolated, aliquoted and stored at $-80^{\circ} \mathrm{C}$ for later use in the MSD multiplex immunoassay.

\section{MSD immunoassay}

The MSD multiplex immunoassay was used to measure the total IgE as well as specific IgE levels. This method is based on the U-plex protocol of MSD (Meso Scale Diagnostics) [20], but was adapted specifically for $\operatorname{IgE}$ measurements, using biotinylated allergens, total IgE capture antibody and IgE standards from Radim Diagnostics (Freiburg im Breisgau, Germany).

The standard curve solutions were prepared on the same day that the plate was run by diluting a stock of total IgE (Radim Diagnostics) with diluent 43 (Meso Scale Diagnostics) to $100,33.33,11.11,3.70,1.23,0.41,0.10$ and $0 \mathrm{IU} / \mathrm{ml}$.

Specific IgEs against either one particular respiratory allergen or a mixture of various respiratory allergens were tested with the MSD assay. Common respiratory allergens were included, as shown in Table 1. A food allergen mixture which can cause anaphylactic reactions with respiratory symptoms was also included (i.e. the mix for nut allergens). The assay is based on the binding of specific IgEs of the serum samples to the biotinylated allergens. The individual allergens and mixtures of allergens were tested on separate plates.

Plate preparation to assess sIgE. To perform the MSD assay, a U-plex 96-well (10-spot) plate was used. Each well contains 10 individual spots and each spot can be linked to a different biomarker with specific linkers (see graphical abstract and MSD U-plex product documentation for more information on the linker binding [20,21]). Per test, one of the 10 spots in each well was used to detect the total IgE, which was necessary to construct the standard curve. It is possible to run a partial plate; however, the protocol described below states the volumes for a full plate. First, nine specific single allergens (to measure sIgEs) and an anti-IgE capture antibody (to quantify total $\operatorname{IgE}$ ) were coupled to one of the 10 different linkers by adding $200 \mu \mathrm{l}$ of the biotinylated allergen/capture antibody to $300 \mu \mathrm{l}$ of the chosen linker containing a streptavidin binding site. All linker-solutions were incubated for $30 \mathrm{~min}$ at room temperature while shaking. Then $200 \mu \mathrm{l}$ stop solution (Meso Scale Diagnostics) was added to prevent further binding and incubated for another $30 \mathrm{~min}$. Afterwards,
$600 \mu \mathrm{l}$ of all 10 linker-allergen/antibody solutions were combined into a single tube. Next, $50 \mu$ of this solution was pipetted into each well to coat the spots on the plate with the appropriate allergen, followed by $1 \mathrm{~h}$ of incubation and the detection of the sIgE levels was performed as described below (Detection of sIgE levels).

Plate preparation to assess sIgE against mixtures of allergens. The preparation of the linkers to assess sIgE against allergen mixtures is very similar to the procedure described above (Plate preparation to assess sIgE), with a few important modifications. To start, only eight spots and thus eight linkers were used: seven for allergen mixtures and one to quantify total IgE. As only eight spots were occupied in plates used to test mixtures of allergens, two spots (spots 5 and 6) were not linked to an allergen, according to the manufacturer's protocol (see graphical abstract). After the 30-min incubation, $600 \mu \mathrm{l}$ of all eight linker-allergen/ antibody solutions were combined into a single tube. As fewer than 10 spots (allergens) were used, extra stop solution was added to gain a total volume of $6 \mathrm{ml}$. All further steps are the same as described above (Plate preparation to assess sIgE).

Detection of sIgE levels. After the 1-h incubation, the plate was washed three times with phosphate-buffered saline-Tween (PBS-T; 0.05\%, $150 \mu \mathrm{l}$ per well), and $25 \mu \mathrm{l}$ of diluent 43 was added to each well. Then $25 \mu \mathrm{l}$ of the corresponding standard series solution or serum sample, containing specific IgEs, was added to the appropriate predetermined wells and incubated overnight at $4^{\circ} \mathrm{C}$. The next day, the plate was washed and $50 \mu \mathrm{l}$ of the custom-made sulfo-tagged detection antibody $(2 \mu \mathrm{g} / \mathrm{ml}$, diluted with diluent 3 from MSD) was added to each well, which completed the immunoassay 'sandwich'. This detection antibody was purchased from Radim Diagnostics and labeled with a sulfo-tag by MSD. After an hour of incubation, the plate was washed again with PBS-T and $150 \mu$ of Read Buffer T was added to each well. Finally, the plate was read within the next 2-5 $\mathrm{min}$ and electrochemiluminescent (ECL) signals (at a wavelength of $620 \mathrm{~nm}$ ) were detected with the MESO QuickPlex SQ 120 device from MSD. Using specific MSD software, values of the ECL signals were generated as output data, which were converted to concentration values in $\mathrm{IU} / \mathrm{ml}$ through calculations with the four-parameter logistic (4PL) standard curve of the total IgE solutions, where IU/ml corresponds to $\mathrm{kU} / \mathrm{l}$ (Supporting information, Fig. S1).

We point out that the total $\operatorname{IgE}$ values obtained via these multiplex MSD assays for each individual sample give an under-estimation of the actual levels. To obtain reliable results for the total IgE, an MSD singleplex assay should be performed. See Supporting Information, Document S2 for a full comparison. 


\section{Data and statistical analyses}

To analyse the results, descriptive statistics were performed using sPss version 25. The linear correlations between the results of the SPT or ImmunoCAP assay and the results of the MSD multiplex immunoassay were evaluated by the Pearson correlation coefficient $(r)$. For the ImmunoCAP assay versus the MSD immunoassay, this was analysed for all data together and per (mix of) allergen. For the SPT versus the MSD immunoassay this was only performed with all data, as for several allergens there was no reaction, resulting in zero values for the SPT that distorted the correlations per allergen. This correlation coefficient was also used to determine the intra- and interassay reproducibility and the difference in incubation time of the MSD multiplex immunoassay. A $P$-value $<0.05$ was regarded statistically significant.

To assess the comparability of the SPT, ImmunoCAP assay and the MSD multiplex immunoassay, a BlandAltman analysis was performed [22]. To compensate for differences in units ( $\mathrm{mm}$ for SPT versus $\mathrm{kU} / \mathrm{l}$ for blood tests) all data were normalized by dividing each individual value over the mean of all values for that method. For all ImmunoCAP tests where $<0.10$ or $<0.35 \mathrm{kU} / \mathrm{l}$ was reported by the medical laboratory, the values were set, respectively, at 0.05 and $0.175 \mathrm{kU} /$ (which is half the detection limit). A Bland-Altman plot is a scatter-plot of the difference between the two methods means ( $y$-axis) against the mean of the two methods ( $x$-axis). The calculated mean difference is shown, and the $95 \%$ confidence limits of agreement are obtained by calculating the mean difference plus or minus twice the standard deviation of the differences.

The sensitivity and specificity of the MSD multiplex immunoassay were determined, both in comparison to the skin prick test and the ImmunoCAP assay of the clinical laboratory. The threshold set for a positive test in both the MSD and ImmunoCAP assay was $0.10 \mathrm{kU} / \mathrm{l}$ for single allergens and $0.35 \mathrm{kU} / \mathrm{l}$ for allergen mixes.

When checking for outliers, subject 3 was eliminated from the analyses: positive scores were observed for all MSD multiplexed measurements (all sIgE levels between 0.3 and $0.7 \mathrm{kU} / \mathrm{l}$ ) and consistent negative scores for the other assays. Moreover, this person reported being diagnosed with essential thrombocythemia, which might explain the divergent results.

\section{Results}

\section{Comparison of the methods}

The results of the MSD multiplex immunoassay were compared to the two most commonly used tests, the
SPT and the ImmunoCAP assay, performed by a clinical laboratory. When an individual scored positive (meaning sensitized) for the allergen it is shown in colour in Fig. 1. When the wheal reaction was observed to be $3 \mathrm{~mm}$ larger than the negative control in the SPT, it was indicated as positive. For the tests of the clinical laboratory (ImmunoCAP) and the MSD immunoassay, a threshold of $0.10 \mathrm{kU} / \mathrm{l}$ for sIgE levels against single allergens or $0.35 \mathrm{kU} / \mathrm{l}$ sIgE against mixes of allergens was used; a result above this threshold was considered positive. Both individual allergens and mixes of allergens were tested in parallel. Most results corresponded well between the three tests, although there were some differences, as discussed later. Looking at the MSD immunoassay, the results of the single allergen tests corresponded better with the clinical symptoms reported by the patients, compared to the results from the tests with the allergen mixes. Corresponding clinical symptoms were reported for each participant, but these data were not taken into account when comparing the SPT, ImmunoCAP and MSD multiplex immunoassay as these methods assess allergic sensitization.

\section{Correlations and Bland-Altman analysis}

The linear correlations, using all data of the different allergens combined, revealed good significant correlations between the results of the MSD multiplex immunoassay and the SPT $(r=0.419 ; P<0.001)$ or the ImmunoCAP assay $(r=0.561 ; P<0.001)$. A slightly lower correlation was observed between the SPT and ImmunoCAP assay $(r=0.353 ; P<0.001)$. The Bland-Altman plots, which determine the comparability of these three methods for all data, are shown in Fig. 2. In all cases, $95 \%$ of the data lie within 2 standard deviations of the mean, as well as there being no clear bias in favor of higher results from one method.

Bland-Altman analyses were also performed at an individual allergen level (Supporting information, Figs S2-S4). Correlations between the MSD multiplex immunoassay and ImmunoCAP assay at an individual allergen level are presented in Table 2. The allergens of cow's milk, Cladosporium herbarum and the mixture of fungi were not included, due to the lack of positives among the tested individuals (none were above $0.10 \mathrm{kU} / \mathrm{l}$ ) for the MSD multiplex immunoassay. For all allergens except house dust mite, significant correlations $(r=0.829-0.978 ; P<0.001)$ were observed.

\section{Sensitivity and specificity}

The results of the MSD multiplex immunoassay were compared to the results of the ImmunoCAP assay and the SPT. The sensitivity and specificity of the MSD multiplex immunoassay were calculated (Table 3). Overall, the 


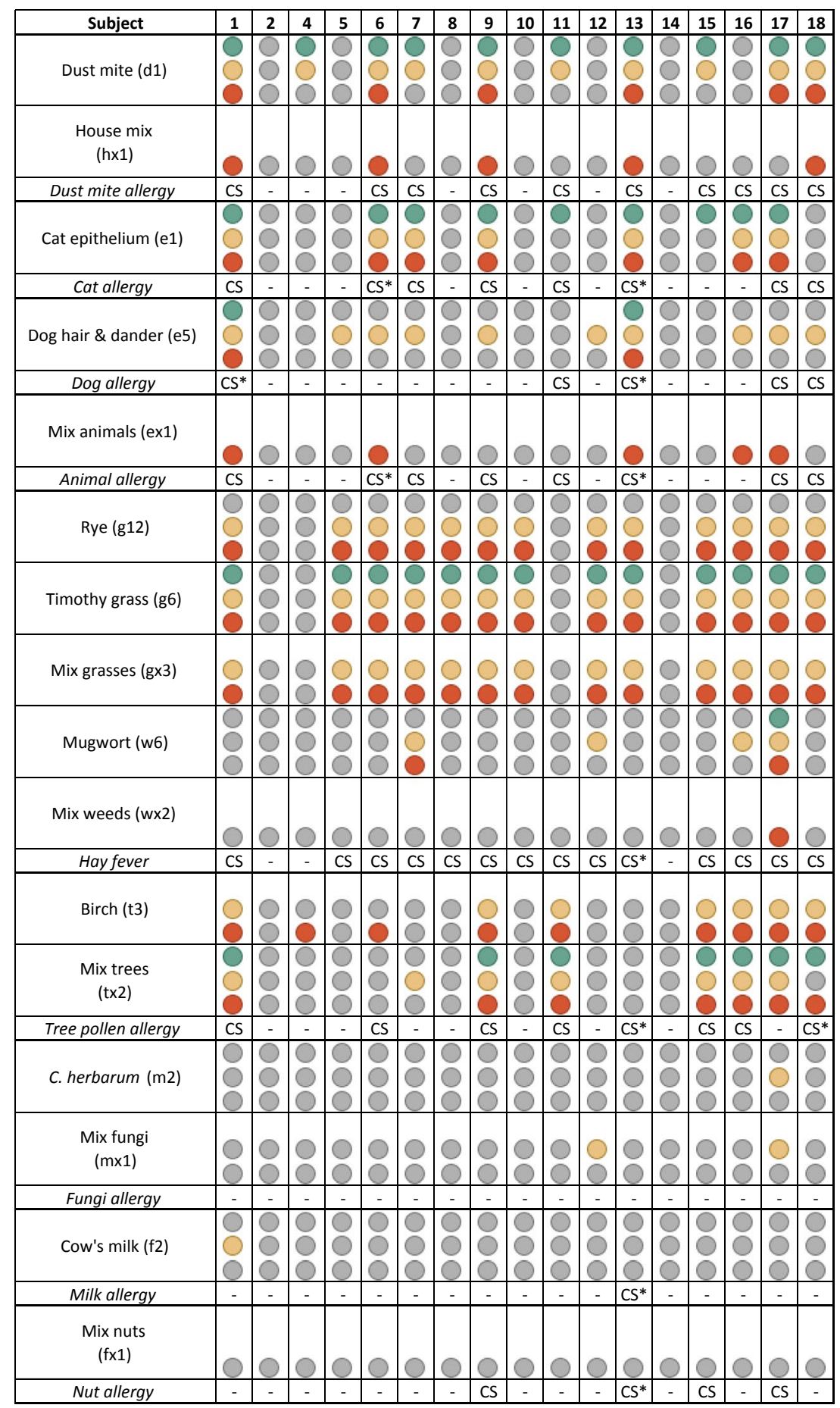

Fig. 1. Results of three methods used to measure the allergy (IgE) levels in 17 individuals. Green: positive result in the skin prick test. Yellow: positive result in the ImmunoCAP assay. Red: positive result in the MSD multiplex immunoassay. Grey: negative result. CS: reported clinical symptoms in the last 12 months. CS ${ }^{*}$ : reported clinical symptoms in the past.

sensitivity of the MSD multiplexed assay was high for sIgEs against most allergens compared to the SPT and ImmunoCAP assay as references ( $\geq 78 \%$ ); however, for some sIgEs the sensitivity values were lower. As described above, sIgE against dust mite allergens was not always detected using MSD (sensitivity $=60 \%$ ). Furthermore, the sensitivity of the MSD assay detecting sIgE against dog epithelium and mugwort allergens was low when using 
(a)
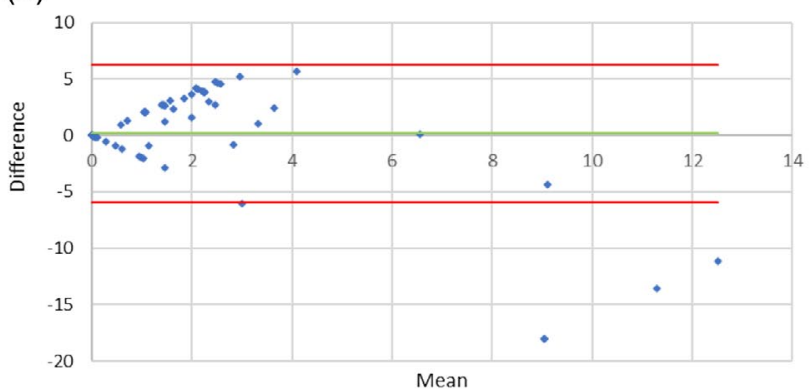

(b)

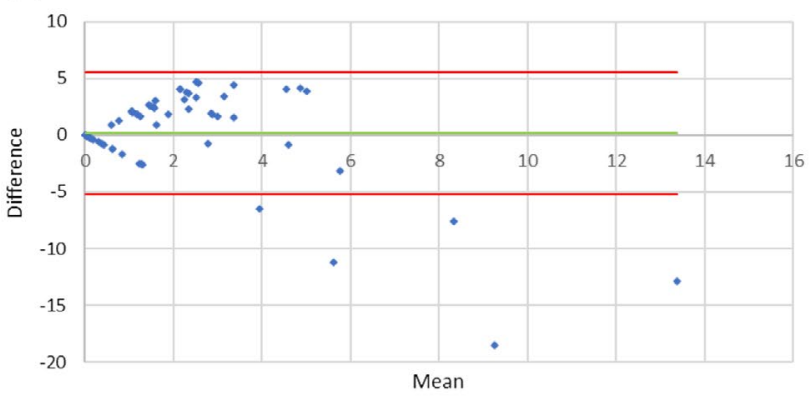

(c)

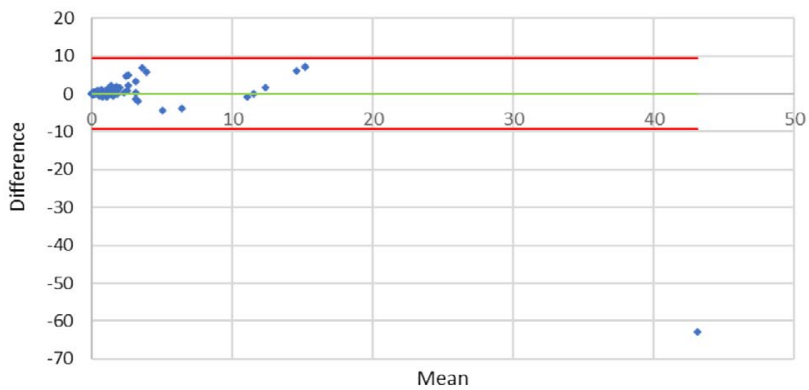

Fig. 2. Bland-Altman plots of the results of all the allergens combined, demonstrating the comparability of (a) the Meso Scale Discovery (MSD) multiplex immunoassay and (b) the ImmunoCAP assay to the skin prick teat (SPT), as well as (c) the MSD multiplex immunoassay compared to the ImmunoCAP assay. The difference between the two methods ( $y$-axis) is plotted against the mean of the two assays ( $x$-axis). The two horizontal red lines represent the $95 \%$ confidence interval of the mean difference.

the ImmunoCAP assay as reference (20-50\%), but was very good (100\%) in comparison with SPT. As the SPT is still used as the gold standard in clinical practice, the MSD multiplex immunoassay detecting sIgE against dog and mugwort allergens was considered sensitive. For some sIgEs, the sensitivity could not be determined due to a lack of positive individuals for these allergens (Fig. 1). When combining the data from all single allergen tests, the sensitivity of the MSD immunoassay was 83 and 76\% in comparison to the SPT and ImmunoCAP assay, respectively. When the data from all the tests were combined,
Table 2. Pearson correlation coefficient $(r)$ and its $P$-value between the results of the ImmunoCAP assay and the Meso Scale Discovery (MSD) multiplex immunoassay

\begin{tabular}{lc}
\hline & Correlation $(r)$ \\
\hline Dust mite & $0 \cdot 271$ \\
Cat & $0 \cdot 829^{* *}$ \\
Dog & $0 \cdot 978^{* *}$ \\
Rye & $0 \cdot 952^{* *}$ \\
Timothy grass & $0 \cdot 904^{* *}$ \\
Birch & $0 \cdot 954^{* *}$ \\
Mugwort & $0 \cdot 976^{* *}$ \\
Mixed grasses & $0 \cdot 969^{* *}$ \\
Mixed trees & $0 \cdot 929^{* *}$ \\
\hline
\end{tabular}

${ }^{*} P$-value $<0.001$ significance level.

the sensitivity of the MSD immunoassay was 86 and $78 \%$ in comparison to the SPT and ImmunoCAP assay, respectively.

In contrast to the sensitivity, the specificity of the MSD multiplex immunoassay was very high ( $\geq 80 \%$ - with the majority showing $100 \%$ specificity). When using the data from the SPT as reference, the only sIgE test with low specificity was rye (24\%). The specificity based on the data from all single allergen tests was 86 and $98 \%$ for the MSD multiplex immunoassay compared to the SPT and ImmunoCAP assay, respectively. Based on all data combined, the specificity was 87 and $97 \%$ for the MSD multiplex immunoassay compared to the SPT and ImmunoCAP assay, respectively.

\section{Reproducibility}

Intra-assay variability. To assess the intra-assay variability of the results of the MSD multiplex immunoassay, each sample was added to the plate in duplicate, for both measurements of sIgE against individual allergens and mixes of allergens. The correlation between the duplicate measures was calculated for all subjects (Table 4). The results show significant positive correlations for all samples $(r=0.95-1$; $P<0.001)$.

Interassay variability. To check the variability of the same samples between two MSD multiplex immunoassays performed on different days, correlations were calculated for sIgE levels of serum samples from seven individuals. The values in Table 4 demonstrate good interassay reproducibility $(r=0.97-1 ; P<0.001)$.

Biological variability over time. For six individuals, results were available of the MSD multiplex immunoassay from a previous blood sampling and testing, in Spring instead of Summer, 1 year and 3 months earlier (Table 4). Plasma samples were used in the prior measurements, while 
Table 3. Sensitivity and specificity of the Meso Scale Discovery (MSD) multiplex immunoassay, comparing the MSD results to the ImmunoCAP assay and skin prick test (SPT). For both the MSD and ImmunoCAP assays, $0 \cdot 10$ and $0.35 \mathrm{kU} / 1$ were set as thresholds for a positive test of single allergens and allergen mixtures, respectively

\begin{tabular}{|c|c|c|c|c|}
\hline & \multicolumn{2}{|c|}{ Sensitivity (\%) } & \multicolumn{2}{|c|}{ Specificity (\%) } \\
\hline & ImmunoCAP & Skin prick test & ImmunoCAP & Skin prick test \\
\hline \multicolumn{5}{|l|}{ Single allergens } \\
\hline Dust mite & 60 & 60 & 100 & 100 \\
\hline Cat & 100 & 78 & 100 & 100 \\
\hline Dog & 20 & 100 & 100 & 100 \\
\hline Cow's milk & n.a. & n.a. & 100 & 100 \\
\hline Rye & 100 & n.a. & 100 & 24 \\
\hline Timothy grass & 100 & 100 & 100 & 100 \\
\hline Cladosporium herbarum & n.a. & n.a. & 100 & 100 \\
\hline Birch & 100 & & 80 & \\
\hline Mugwort & 50 & 100 & 100 & 94 \\
\hline For all single allergens combined & 76 & 83 & 98 & 86 \\
\hline \multicolumn{5}{|l|}{ Allergen mixtures } \\
\hline Mixed grasses & 100 & & 100 & \\
\hline Mixed fungi & n.a. & & 100 & \\
\hline Mixed trees & 86 & 100 & 90 & 100 \\
\hline Total for all allergen extracts & 78 & 86 & 97 & 87 \\
\hline
\end{tabular}

n.a. $=$ not applicable.

Table 4. Pearson correlation coefficients and their $P$-values for comparison of results within one assay (intra-assay reproducibility) and between assays over time (interassay reproducibility), as well as for biological variability over time (two different sampling times) and differences in incubation times (overnight versus $2 \mathrm{~h}$ incubation) of the Meso Scale Discovery (MSD) multiplex immunoassay

\begin{tabular}{|c|c|c|c|c|}
\hline Subject & Intra-assay reproducibility & Interassay reproducibility & Biological variability over time & Difference in incubation time \\
\hline 1 & $1.000^{\star *}$ & $0.998^{* *}$ & $0 \cdot 976^{* *}$ & $0.958^{* *}$ \\
\hline 2 & $0.996^{* *}$ & $1.000^{\star *}$ & $0.994^{* *}$ & $1.000^{* *}$ \\
\hline 3 & $0.994^{\star *}$ & & & $0.996^{* *}$ \\
\hline 4 & $0.997^{* *}$ & & & $1.000^{* *}$ \\
\hline 5 & $0.999^{* *}$ & & & $0.989^{* *}$ \\
\hline 6 & $1.000^{* *}$ & $0.997^{* *}$ & $0.943^{* *}$ & $0.995^{* *}$ \\
\hline 7 & $0.990^{* *}$ & $1.000^{\star *}$ & $1.000^{* *}$ & $1 \cdot 000^{* *}$ \\
\hline 8 & $0.986^{* *}$ & & & $0.948^{* *}$ \\
\hline 9 & $0.999^{* *}$ & & $0.962^{* *}$ & $0.998^{* *}$ \\
\hline 10 & $0.981^{\star *}$ & & & $0.966^{* *}$ \\
\hline 11 & $0.996^{* *}$ & $0 \cdot 979^{*}$ & & $0.993^{* *}$ \\
\hline 12 & $1.000^{* *}$ & & & $1.000^{* *}$ \\
\hline 13 & $0.997^{* *}$ & $0.996^{\star *}$ & $0.652^{\star}$ & $0.999^{* *}$ \\
\hline 14 & $0.992^{* *}$ & & & $0.997^{\star *}$ \\
\hline 15 & $0.996^{* *}$ & & & $0.925^{* *}$ \\
\hline 16 & $0.999 * *$ & $0.970^{* *}$ & & $0.999 * *$ \\
\hline 17 & $0.945^{* *}$ & & & $0.913^{* *}$ \\
\hline 18 & $1.000^{* *}$ & & & $0.998^{* *}$ \\
\hline
\end{tabular}

${ }^{*} P$-value $<0.001$ significance level; ${ }^{*} P$-value $<0.05$ significance level.

serum samples were analysed in the current tests. However, previous analysis of serum and plasma samples collected at the same time showed a correlation of $r=0.92(P<0.001)$, indicating that both matrices show comparable sIgE levels with the MSD multiplex immunoassay. The biological variation over time was observed to be consistent $(r=0.94-1 ; P<0.001)$. The correlation for subject 13 was, however, lower $(r=0.65 ; P<0.05)$.

Comparison incubation overnight or $2 h$. The MSD multiplex immunoassay protocol states that after adding the standard series and samples, either an incubation overnight 
at $4^{\circ} \mathrm{C}$ or for $2 \mathrm{~h}$ at room temperature while shaking the plate can be applied. To compare the two different incubation periods, the assay was performed in both ways for the individual allergens (Table 4). The two variations of the assay showed high and statistically significant positive correlations $(r=0.91-1 ; P<0.001)$.

\section{Discussion}

This is the first study, to our knowledge, describing a comparison between the MSD multiplex immunoassay and the well-established SPT and ImmunoCAP assay to detect sIgE levels. Overall, the performance of the newly developed MSD multiplex immunoassay for sIgE was comparable to the SPT and ImmunoCAP assay, as observed from correlations and Bland-Altman plots. It also showed high sensitivity and specificity compared to the SPT and ImmunoCAP assay. Furthermore, low inter- and intra-assay variation and high reproducibility was observed.

It is important to note that the presence of $\operatorname{sigE}$ reflects allergic sensitization and not necessarily clinical allergy. As the purpose of this study was to compare various methods to test allergic sensitization, the reported clinical allergy symptoms were not included in the analysis. For the majority of the patients, the results of the allergic sensitization match their clinical symptoms, while for some it does not. This inconsistency can be explained by multiple factors, including the patients' ages, duration of allergen avoidance at the time of testing, selection of patients, occasionally undetectable sIgE levels and clinical disorders of patients being studied [9]. In the current study, this inconsistency can be observed, for instance, for the ImmunoCAP assay, which showed more positive cases for sIgE against dog allergens than cases reporting allergy against dogs, while the corresponding results of the SPT and MSD multiplex immunoassay were negative (Fig. 1). This may suggest that these individuals (six of 18) were sensitized without clinical signs of allergy, or that the ImmunoCAP assay for $\mathrm{d} 1$ is less specific compared to the SPT and MSD immunoassay. The low specificity observed for the sIgE test against rye in the MSD multiplex immunoassay compared to the SPT was probably due to cross-reactivity of $\operatorname{IgE}$ antibodies against timothy grass and rye, as both species of grasses belong to the Poaceae family [23]. The cross-reactivity caused a person allergic to timothy grass to also test positive for $\operatorname{sgE}$ against rye in the MSD multiplex immunoassay and the ImmunoCAP assay. However, the SPT showed only an allergic sensitization to timothy grass (Fig. 1).

Based on the Bland-Altman plots, the current study shows acceptable similarity between the three tested methods to assess allergic sensitization, especially between the
MSD multiplex immunoassay and the ImmunoCAP assay - two methods testing for sIgE levels (Fig. 2c). One value was outside the $95 \%$ confidence limits, which might have been due to an over-estimation in the MSD multiplex immunoassay, as the sIgE levels of the sample were outside the standard curve (i.e. 647.91 kU/1 for MSD immunoassay and $61.90 \mathrm{kU} / 1$ for the ImmunoCAP assay). Regarding comparisons against the SPT, the Bland-Altman plots show a funnel-like shape (Fig. 2a,b), which indicates that the variation of at least one method depends strongly upon the magnitude of measurements. This is probably due to the zero values for negative SPT versus continuous low levels for the blood tests. In all cases, the mean difference between the methods are close to zero and $95 \%$ of the data lie within 2 standard deviations of the mean, indicating very low bias. When looking at an individual allergen level, no correlation was observed for house dust mite, and it showed also the least comparability to the ImmunoCAP assay according to the corresponding BlandAltman plot (Supporting information, Fig. S2). Regarding the other allergens, good agreements were observed with the SPT and ImmunoCAP assay, as shown by the correlations (Table 2) and in the corresponding Bland-Altman plots (Supporting information, Figs S2-S4).

In previous studies, the sensitivity and specificity of the ImmunoCAP assay were compared to the gold standard, the SPT [24-26]. For a select set of allergens that were analysed both in those previous studies with the ImmunoCAP assay and with the MSD multiplex immunoassay in the current study, similar sensitivity and specificity were observed (Supporting information, Table S3). For the detection of sIgE against timothy grass, cats and dogs, the MSD multiplex immunoassay showed higher sensitivity and specificity values than the ImmunoCAP assay when compared to the SPT. The specificity values related to dust mite and $C$. herbarum were also higher for the MSD multiplex immunoassay; however, the sensitivity for dust mite and the specificity for rye were higher in the ImmunoCAP assay.

This study is subject to some limitations, including the fact that results of only three mixtures of allergens from the ImmunoCAP assay were available and could be compared, and only one mixture was available for parallel tests with the SPT (Table 1). To know with certainty if the sensitivity and specificity of the MSD multiplex immunoassay with allergen mixes are the same as (or less than) when using single allergens requires more extensive comparisons. Furthermore, the sample size was small, as this was a pilot study and participants were selected based on their self-reported allergies. Additionally, the three allergy tests were performed with allergens from different suppliers (e.g. biotinylated allergen extracts could not be used for SPT and vice versa), which makes the results 
less comparable. Nevertheless, the sensitivity and specificity of the MSD multiplex immunoassay were overall quite high.

Moreover, our results with the MSD multiplex immunoassay show very low inter- and intra-assay variability. The assay also showed to be reproducible, as samples tested in Spring and Summer (1 year and 3 months between sampling times) showed consistent results. A lower yet still significant correlation was observed only for subject $13(r=0.65 ; P<0.05)$, which was most probably due to seasonal effects. Additionally, a more rapid version of the assay, only adopting a 2 -h incubation time, produced very similar results compared to an overnight incubation ( $r=0.91-1 ; P<0.001)$.

To conclude, the multiplex immunoassay from Meso Scale Diagnostics, combined with standards and biotinylated allergens from Radim Diagnostics, allowed highthroughput analysis of specific IgEs against respiratory allergens in serum samples. The results were reproducible and also comparable to the commonly used, well-established methods (SPT and ImmunoCAP assay). The MSD multiplex immunoassay needs small blood sample volumes and is also very versatile: both individual allergens as well as mixes of allergens can be analysed. The choice of allergens can be adapted easily to the patients' or researchers' needs. Its ease in use makes it applicable in both clinical and research settings.

\section{Acknowledgements}

We thank our study volunteers for participating in this study. S. L was the beneficiary of a post-doctoral fellowship (12L5216N) provided by the Research FoundationFlanders (FWO) and the Flemish Institute of Technological Research (VITO). The work was funded by an FWO Research Grant (1523817N).

\section{Disclosures}

The authors declare that there are no conflicts of interest.

\section{Author contributions}

S. L. and G. K. conceived and designed the experiments. S. L., I. W., J. M. and G. S. recruited volunteers and processed samples. M. R. performed the skin prick test and venepuncture. J. M., I. W. and S. L. performed the experiments. J. M., S. L. and I. W. analysed the data. S. L., G. K. and M. R. contributed the reagent, materials and analysis tools. J. M., I. W. and S. L. wrote the paper. All authors evaluated and approved the manuscript.

\section{Data Availability Statement}

The data that support the findings of this study are available from the corresponding author upon reasonable request.

\section{References}

1 World Health Organization (WHO). Global surveillance, prevention and control of chronic respiratory diseases: a comprehensive approach. Geneva, Switzerland: WHO, 2007.

2 World Health Organization (WHO). Asthma Fact Sheet. Geneva, Switzerland: WHO, 2013.

3 Blomme K, Tomassen P, Lapeere $\mathrm{H}$ et al. Prevalence of allergic sensitization versus allergic rhinitis symptoms in an unselected population. Int Arch Allergy Immunol 2013; 160:200-7.

4 Akdis CA, Hellings PW, Agache I. Global atlas of allergic rhinitis and chronic rhinosinusitis. Zurich: European Academy of Allergy and Clinical Immunology, 2015.

5 Pawankar R, Holgate ST, Canonica GW, Lockey RF, Blaiss MS. WAO White Book on Allergy: Update 2013 - Executive Summary. Milwaukee, WI: World Allergy Organization, 2013.

6 Sciensano. Health Interview Survey. Brussels, Belgium: Sciensano, 1997-2018.

7 Peveri S, Pattini S, Costantino MT et al. Molecular diagnostics improves diagnosis and treatment of respiratory allergy and food allergy with economic optimization and cost saving. Allergol Immunopathol 2019; 47:64-72.

8 Migueres M, Dávila I, Frati F et al. Types of sensitization to aeroallergens: definitions, prevalences and impact on the diagnosis and treatment of allergic respiratory disease. Clin Transl Allergy 2014; 4:16.

9 Boyce JA, Assa'ad A, Burks AW et al. Guidelines for the diagnosis and management of food allergy in the United States: report of the NIAID-sponsored expert panel. J Allergy Clin Immunol 2010; 126:S1-S58.

10 Bousquet J, Khaltaev N, Cruz AA et al. Allergic Rhinitis and its Impact on Asthma (ARIA) 2008 update (in collaboration with the World Health Organization, GA(2)LEN and AllerGen). Allergy 2008; 63(Suppl 86):8-160.

11 van Hage M, Hamsten C, Valenta R. ImmunoCAP assays: pros and cons in allergology. J Allergy Clin Immunol 2017; 140:974-7.

12 Wide L, Bennich H, Johansson SGO. Diagnosis of allergy by an in-vitro test for allergen antibodies. Lancet 1967; 290:1105-7.

13 Cox L, Williams B, Sicherer S et al. Pearls and pitfalls of allergy diagnostic testing: report from the American College of Allergy, Asthma and Immunology/American Academy of Allergy, Asthma and Immunology Specific IgE Test Task Force. Ann Allergy Asthma Immunol 2008; 101:580-92.

14 Gendo K, Larson EB. Evidence-based diagnostic strategies for evaluating suspected allergic rhinitis. Ann Intern Med 2004; 140:278-89. 
15 Tighe PJ, Ryder RR, Todd I, Fairclough LC. ELISA in the multiplex era: potentials and pitfalls. Proteomics Clin Appl 2015; 9:406-22.

16 Eigenmann PA, Kuenzli M, D’Apuzzo V et al. The ImmunoCAP Rapid Wheeze/Rhinitis Child test is useful in the initial allergy diagnosis of children with respiratory symptoms. Pediatr Allergy Immunol 2009; 20:772-9.

17 Jensen E, Weber SB. ImmunoCAP rapid - a near-patient test for qualitative detection of specific $\operatorname{IgE}$ antibodies against inhalation allergens in human whole blood manufactured by Phadia AB, now Thermo Fisher Scientific Inc, Uppsala, Sweden. Report from an evaluation under standardised and optimal conditions and in primary health care organised by SKUP Evaluated at the request of Phadia. Denmark: SKUP, 2013.

18 Labco Quality Diagnostics. ISAC Test: Simultaneous assessment of 112 allergenic proteins. Munich, Germany: Labco Quality Diagnostics, 2013.

19 Asher MI, Keil U, Anderson HR et al. International Study of Asthma and Allergies in Childhood (ISAAC): rationale and methods. Eur Respir J 1995; 8:483-91.

20 Meso Scale Diagnostics. U-plex development packs: for the development of multiplex assays 2018. Available at: https://www. mesoscale.com/ /media/files/product\%20inserts/u-plex\%20dev elopment\%20pack.pdf (accessed 23 December 2019).

21 Meso Scale Diagnostics. U-plex - personalized multiplexing 2020. Available at: https://www.mesoscale.com/\%7E/media/files/ product\%2520s/u-plex\%2520platform\%2520.pdf (accessed 23 December 2019).

22 Giavarina D. Understanding Bland Altman analysis. Biochem Med 2015; 25:141-51.

23 Schenk S, Breiteneder H, Susani M et al. T-cell epitopes of Phl p 1, major pollen allergen of timothy grass (Phleum pratense): evidence for crossreacting and non-crossreacting T-cell epitopes within grass group I allergens. J Allergy Clin Immunol 1995; 96:986-96.

24 Calabria CW, Dietrich J, Hagan L. Comparison of serum-specific $\mathrm{IgE}$ (ImmunoCAP) and skin-prick test results for 53 inhalant allergens in patients with chronic rhinitis. Allergy Asthma Proc 2009; 30:386-96.

25 Ewan PW, Coote D. Evaluation of a capsulated hydrophilic carrier polymer (the ImmunoCAP) for measurement of specific IgE antibodies. Allergy 1990; 45:22-9.

26 Griffiths RLM, El-Shanawany T, Jolles SRA et al. Comparison of the performance of skin prick, ImmunoCAP, and ISAC tests in the diagnosis of patients with allergy. Int Arch Allergy Immunol 2017; 172:215-23.

\section{Supporting Information}

Additional supporting information may be found in the online version of this article at the publisher's web site:

Table S1. Comparison of the characteristics of the multiplex ISAC assay and the MSD multiplex immunoassay. van Hage M, Hamsten C, Valenta R. ImmunoCAP assays: Pros and cons in allergology. J Allergy Clin Immunol. 2017;140(4):974-7.

Table S2. Comparison of the sensitivity and specificity of the ImmunoCAP assay assessed in previous research and the MSD multiplex immunoassay, all compared to the SPT.

Fig. S1. An example of a 4PL (four-parameter logistic) curve. The equation of this standard curve was used to calculate the concentrations of the samples $(\mathrm{x})$ in IU/mL. With $y$ : the detected ECL signal per sample/standard; b1: value of Calc. Top; b2: value of Calc. Bottom; b3: value of Calc. MidPoint; b4: Calc. Hillslope.

Fig. S2. Bland-Altman plots of the different allergens or mixes of allergens, demonstrating the comparability of the MSD multiplex immunoassay to the ImmunoCAP ${ }^{\mathrm{TM}}$ assay. The difference between the two methods ( $\mathrm{y}$-axis) is plotted against the mean of the two assays ( $\mathrm{x}$-axis). The two horizontal red lines represent the $95 \%$ confidence interval of the mean difference.

Fig. S3. Bland-Altman plots of the different allergens or tree mix of allergens, demonstrating the comparability of the MSD multiplex immunoassay to the SPT. The difference between the two methods ( $y$-axis) is plotted against the mean of the two assays (x-axis). The two horizontal red lines represent the $95 \%$ confidence interval of the mean difference.

Fig. S4. Bland-Altman plots of the different allergens or tree mix of allergens, demonstrating the comparability of the ImmunoCAP ${ }^{\mathrm{TM}}$ assay to the SPT. The difference between the two methods (y-axis) is plotted against the mean of the two assays (x-axis). The two horizontal red lines represent the $95 \%$ confidence interval of the mean difference. 